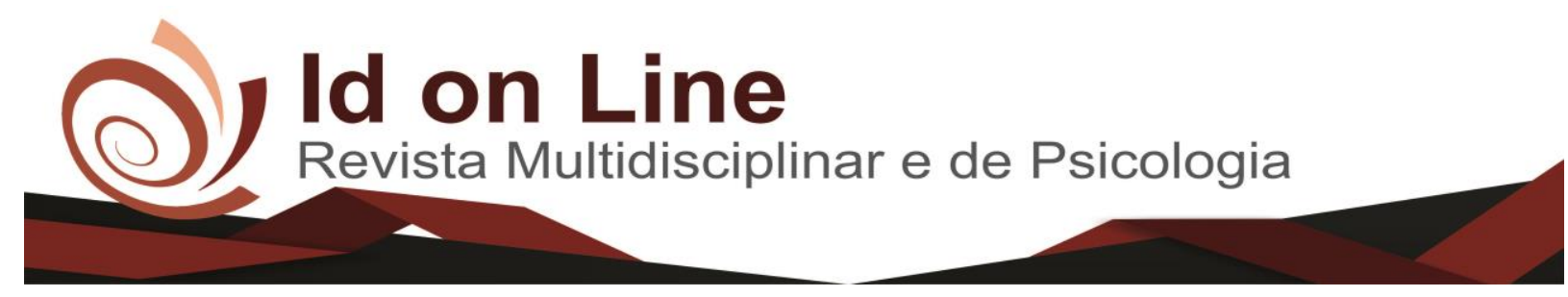

Artigo

\title{
Timed Up and Go em Idosos Residentes na Comunidade
}

Rubiele Almeida Prado ${ }^{1}$, Kleyton Trindade Santos ${ }^{2}$, Luciana Araújo dos Reis ${ }^{3}$, Karla Cavalcante Silva de Morais ${ }^{4}$, Andressa Porto Dutra ${ }^{1}$, Sabrina Almeida Porto ${ }^{1}$.

\begin{abstract}
Resumo: Objetivo: avaliar os idosos segundo o Timed Up And Go verificando o tempo médio e classificação do risco de quedas. Materiais e Métodos: estudo transversal, descritivo, vinculado à pesquisa intitulada "Quedas e fatores associados em idosos", aprovada através do CAAE: 62170516.4.0000.5578. Foi realizada em uma Unidade de Saúde da Família, no município de Vitória da Conquista/BA, e contou com uma amostra composta por 45 idosos. Os participantes responderam um questionário sociodemográfico, acrescido do Mini Exame do Estado Mental, e aplicação do Timed Up and Go. Resultados: a maioria dos idosos era do sexo feminino, e 51,1\% apresentaram moderado risco de quedas. O tempo de realização do teste foi menor para homens e grupo etário de 60-69 anos. Conclusão: conclui-se que mais da metade dos idosos avaliados apresentaram moderado risco de quedas e que as mulheres e indivíduos acima dos 70 anos apresentam uma média maior de tempo para a realização do teste.
\end{abstract}

Palavras-Chave: Acidentes por queda; Idoso; Locomoção.

\section{Timed Up and Go in Elderly Community Residents}

\begin{abstract}
Objective: To evaluate the elderly according to the Timed Up And Go verifying the average time and classification of the risk of falls. Materials and Methods: a cross-sectional, descriptive study, linked to the research entitled "Associated falls and factors in the elderly", approved through the CAAE: 62170516.4.0000.5578. It was performed at a Family Health Unit, in the city of Vitória da Conquista, Bahia, and had a sample composed of 45 elderly. Participants answered a sociodemographic questionnaire, plus the Mini Mental State Examination and Timed Up and Go application. Results: the majority of the elderly were female, and $51.1 \%$ presented moderate risk of falls. The time of the test was shorter for men and age group of 60-69 years. Conclusion: it is concluded that more than half of the elderly evaluated had a moderate risk of falls and that women and individuals over 70 years of age present a longer average time to perform the test.
\end{abstract}

Keywords: Accidents by fall; Elderly; Locomotion.

\footnotetext{
${ }^{1}$ Graduanda em Fisioterapia, Faculdade Independente do Nordeste-FAINOR, Vitória da Conquista, Bahia, Brasil.

E-mail: rubi-prado@hotmail.com

2 Fisioterapeuta, Mestre em Ciências da Saúde, Docente da Faculdade Independente do Nordeste-FAINOR, http://www.fainor.com.br, Vitória da Conquista, Bahia, Brasil.

3 Fisioterapeuta, Doutora em Ciências da Saúde, Docente da Faculdade Independente do Nordeste-FAINOR, http://www.fainor.com.br, Vitória da Conquista, Bahia, Brasil.

4 Fisioterapeuta, Mestre em Saúde Coletiva, Docente da Faculdade Independente do Nordeste-FAINOR, http://www.fainor.com.br, Vitória da Conquista, Bahia, Brasil.

Autor correspondente: Rubiele Almeida Prado, e-mail: rubi-prado@ hotmail.com. Telefone: (77) 9.8829-6014
} 


\section{Introdução}

O envelhecimento é um fenômeno biológico, social e psicológico que alcança todo ser humano na indenidade de sua vivência, alterando seu estado físico e mental, gerando em alguns casos eventos desfavoráveis a saúde (GASPAROTTO; FALSARELLA; COIMBRA, 2014). A cada dia vem ocorrendo um aumento significativo de pessoas idosas no mundo e estima-se que em 2025 essa população chegue a 800 milhões de idosos acima de 65 anos de idade, em 2030 a presunção é de 40,7 milhões no Brasil (SILVA et al., 2017).

Dentre os problemas que merecem atenção à saúde do idoso, a queda apresenta-se com grande importância devido a seu poder de morbimortalidade. A queda é um resultado de fatores ambientais, biomédicos, fisiológicos e psicossociais que colocam em risco a risco a estabilidade do indivíduo. (MELO; SANTOS; GRATÃO, 2014). Estima-se que atualmente 28 a 35\% dos idosos acima de 65 anos caem uma vez por ano, e $49 \%$ acima de 70 anos, sendo que 10,8\% referiram duas ou mais quedas (GASPAROTTO; FALSARELLA; COIMBRA, 2014).

Na busca por diagnosticar parâmetros clínicos preditores do risco de queda em idosos, existem métodos de avaliação funcional. E dentre eles se destaca o Timed Up and Go (TUG), o qual apresenta bons resultados em relação ao equilíbrio. O TUG é um teste criado para avaliar a funcionalidade e o risco de quedas em idosos que foi originalmente descrito por Podsiadlo e Richardson (1991). O teste Timed "Up and Go" (também conhecido como TUG) tem apresentado bons resultados ao ser empregado como teste de equilíbrio, pois envolve movimento funcional. Este teste pode ser definido como uma proporção sensível e específica para fazer uma discriminação entre idosos que podem cair e não cair. O TUG tem como princípio medir o tempo que uma pessoa pode levar para realizar alguns movimentos funcionais, tais como, levantar-se de uma cadeira, caminhar, dar uma volta, voltar ao ponto inicial e sentar-se (COSTA; SOUSA, 2016).

Diante dessas definições, é importante salientar que a queda do idoso é de grande importância para a saúde pública. Pois, atualmente, está ocorrendo um crescimento elevado nessa população, e com isso faz-se necessário estudar melhor os riscos que podem ocasionar quedas e idosos. Sendo assim, se torna necessário conhecer melhor a proporção deste evento, e como acontece e como é conduzido, para assim analisar medidas adotadas, reorganizandoas de forma a obter uma maior adequabilidade e efetividade dos serviços prestados pelos 
profissionais de saúde. Este estudo tem por objetivo avaliar o risco de quedas em idosos através do TUG.

\section{Materiais e Métodos}

Trata-se de um estudo transversal, descritivo, de caráter quantitativo, vinculado à pesquisa intitulada "Quedas e fatores associados em idosos", realizada em uma Unidade de Saúde da Família, no município de Vitória da Conquista/BA.

Atualmente a cidade conta com 07 Unidades Básicas de Saúde tradicionais que ofertam serviços básicos e gratuitos para a população. A escolha da Unidade de Saúde aos quais os pesquisadores se reportaram para investigação se deu através de sorteio, a fim de minimizar possível viés de direcionamento da pesquisa.

A população do estudo foi representada 45 idosos, cadastrados na Unidade Básica de Saúde selecionada pelos pesquisadores para realização da coleta, que atenderam os critérios de inclusão adotados: apenas os idosos que obtiveram estado cognitivo preservado, testado a partir da aplicação prévia do mini-exame da saúde mental (Mini-Mental) (FOLSTEIN; McHUGR, 1979). Foram excluídos do estudo aqueles idosos que forem incapazes de compreender as perguntas, mesmo que apresentassem um estado cognitivo adequado de acordo com o Mini- Mental.

Para investigação dos dados foi utilizado um questionário, composto por variáveis sociodemográficas, Mini Exame do Estado Mental (FOLSTEIN; McHUGR, 1979), e aplicação do Timed Up and Go (PODSIADLO, RICHARDSON, 1991). A coleta ocorreu em uma única etapa, sendo que os pesquisadores abordavam os idosos na Unidade Básica de Saúde, enquanto os mesmos aguardavam para realização de consultas ou outros procedimentos, e aplicavam o questionário e o teste.

A pesquisa seguiu todos os princípios da resolução 466/12 e contou com a aprovação do Comitê de Ética em Pesquisa da Faculdade Independente do Nordeste (FAINOR), com o parecer consubstanciado de número de CAAE: 62170516.4.0000.5578.

Os dados da pesquisa foram tabulados e analisados no programa estatístico SPSS 22.0, onde foram calculadas as freqüências relativas e absolutas de todas as variáveis de interesse. 


\section{Resultados}

A partir dos resultados apresentados na tabela 1, pode-se avaliar o perfil sociodemográfico da população observando uma maior frequência de idosos avaliados do sexo feminino (64,4\%), com companheiros (as) (53,3\%), idosos de 60 a 69 anos (60\%), e idosos acima de $70(40 \%)$.

Tabela 1. Perfil sociodemográfico dos idosos. Vitória da Conquista/BA, 2017.

\begin{tabular}{lcc}
\hline Variáveis & n & \% \\
\hline Sexo & & \\
Masculino & 16 & 35,6 \\
Feminino & 29 & 64,4 \\
Estado Civil & & \\
Sem companheiro (a) & 21 & 46,7 \\
Casado (a) & 24 & 53,3 \\
Grupo etário & & \\
$60-69$ anos & 27 & 60 \\
70 anos ou mais & 18 & 40 \\
\hline Total & 45 & 100,0 \\
\hline
\end{tabular}

Fonte: Dados da pesquisa.

No estudo foi verificada a média geral de realização do TUG por sexo e grupo etário. Através da tabela 2 é possível notar que os homens e grupo etário de 60-69 anos tiveram uma média de realização do teste melhores que os seus grupos equivalentes.

Tabela 2. Média geral do tempo da realização do TUG. Vitória da Conquista/BA, 2017.

\begin{tabular}{ll}
\hline Variáveis & Média de realização do teste \\
\hline Sexo & \\
Masculino & 10,11 \\
Feminino & 10,39 \\
Grupo etário & \\
60-69 anos & 9,54 \\
70 anos ou mais & 11,41 \\
\hline Média geral & 10,29 \\
\hline
\end{tabular}

Fonte: Dados da pesquisa. 
A tabela 3 apresenta informações referentes ao risco de quedas nos idosos avaliados através do TUG. Observa-se que há uma quantidade maior de pessoas que apresenta um moderado risco de quedas.

Tabela 3. Classificação do risco de quedas em idosos. Vitória da Conquista, Bahia, Brasil, 2017.

\begin{tabular}{lcc}
\hline \multicolumn{1}{c}{ Variáveis } & n & \% \\
\hline Classificação do Score do TUG & & \\
Baixo Risco de Quedas & 21 & 46,7 \\
Moderado Risco de Quedas & 23 & 51,1 \\
Alto risco de Quedas & 1 & 2,2 \\
\hline Total & 45 & 100 \\
\hline
\end{tabular}

Fonte: Dados da pesquisa.

\section{Discussão}

O perfil sociodemográfico encontrado na pesquisa evidenciou que o gênero que mais predomina nos idosos é o sexo feminino $(64,4 \%)$, que correlaciona com estudo de Presta et al. (2011), onde a amostra estudada também apresentou uma predominância no gênero feminino, e isso devido a mortalidade ser maior em homens por estar expostos a acidentes e profissões de risco.

Em outro estudo de Luz et al. (2014) explica que esses achados podem ser justificados, pois nota-se que nos últimos anos a expectativa de vida aumentou consideravelmente, principalmente no sexo feminino, devido apresentar uma mortalidade menor se comparada ao sexo masculino, sendo explicada por maiores cuidados e procura aos serviços de saúde, uma prevalência menor de fatores que resultam em óbitos, tanto por fatores internos como doenças circulatórias, doenças relacionadas ao alcoolismo e ao tabagismo, como por causas externas, como assassinatos e atropelamentos, que normalmente apresenta maior índice na população masculina.

Ao avaliar o tempo de realização do TUG, foi possível verificar que os homens apresentaram tempo médio menor que o das mulheres, embora a diferença fosse pequena. De acordo com o estudo de Leitão et al. (2000), identifica-se um menor consumo máximo de oxigênio em mulheres quando comparada aos homens, além do mais, as mulheres tem uma menor capacidade de transportar oxigênio, essas condições fazem com que o desempenho 
esportivo seja menor nas mulheres, de 6 a 15\% em comparação aos homens, mesmo que a capacidade de adaptação do treinamento seja semelhante. Isso justifica a atual pesquisa ter apresentado uma média maior nas mulheres do que nos homens para a realização do teste TUG, sendo a média de 10,39 s, enquanto comparada aos homens, tendo um tempo gasto de 10,11 para a efetuação do teste.

No presente estudo também foi observado a média por grupo etário, sendo dividido em 60-69 anos e 70 anos ou mais. Verificou-se que o primeiro grupo apresentou quase 2 segundos a menos em sua média, se comparado ao grupo dois, sugerindo que as pessoas com idade mais elevada necessitam de mais tempo para a realização do TUG. Esses achados se assemelham ao estudo de Silva et al. (2016) que encontrou valores de 11,91 segundos para o grupo de 60-69 anos e 13,47s para o grupo de 70 anos ou mais.

Wamser et al. (2015), também considerou o TUG para grupo etários e verificou valores de 8,1s para o grupo de 60-69 anos, 9,2s para o grupo de 70 a 79 anos e 11,3s para os idosos com 80 anos ou mais. Todos esses achados reforçam a ideia de um maior comprometimento na velocidade da marcha com a evolução da idade, fazendo com que o idoso merece maior cuidado.

Ansai et al. (2014), destacam que os idosos que apresentarem menos desempenho nos testes de caminhada estão mais propícios e exposto a sofrer quedas, devido a sua condição física que condiciona para uma marcha mais lenta, e esses achados ficam mais evidentes e se tornam mais preocupantes com o avançar da idade.

Ao proceder com a avaliação do risco de queda na amostra de acordo com o tempo apresentado no teste, observou-se que $46,7 \%$ dos idosos tiveram baixo risco, $51,1 \%$ risco moderado, e apenas 2,2\% com alto risco. Esses dados encontrados corroboram com um estudo realizado por Bretan et al. (2013), com idosos que frequentavam o ambulatório de uma Unidade Básica de Saúde, onde o moderado risco de quedas atingiu um maior número de idosos $(63,72 \%)$

Abreu et al. (2015), traz que um moderado risco de quedas repercute diretamente em seu físico, podendo vir a trazer consequências graves como lesões, fraturas, hematomas. Além de que, pode gerar transtornos psicológicos aos familiares e também gastos extras a saúde pública com maiores períodos de internamento e tratamento, isso exige uma série de cuidados utilizando mais serviços e gerando prejuízos.

Segundo Alves et al. (2016) as quedas representam um dos principais problemas clínicos observados na população idosa, gerando uma série de efeitos negativos tanto para os familiares 
quanto para a saúde pública, levando o idoso a dependência funcional e/ou deficiência, sendo necessária a utilização de mais serviços e maiores gastos.

Diante dos resultados encontrados observa-se que é de extrema importância o rastreio do risco de quedas na população idosa, para que sejam adotadas medidas preventivas, evitando todo o ciclo de complicações referentes a ocorrência desse evento.

As limitações da pesquisa foram em relação ao tamanho da amostra, devido ter sido entrevistado somente 45 idosos de apenas uma de várias Unidades de Saúde da Família localizadas na Cidade. Outra limitação apontada é o delineamento transversal e apenas descritivo do estudo, o que impede realização de causa/efeito. Entretanto, é relevante ressaltar que o uso TUG é importante para avaliar a funcionalidade e o risco de quedas na população de idade mais avançada.

Vale também destacar que o estudo apresenta-se com extrema aplicabilidade clínica, por avaliar o risco de quedas através de um instrumento simples e validado na população idosa, podendo servir de alerta para condutas preventivas, evitando esse evento desfavorável e consequentemente problemas de saúde que possam vir a surgir decorrentes delas.

\section{Conclusão}

Foi possível concluir que mulheres e idosos com idades mais avançadas tendem a apresentar a ter uma marcha mais lenta, aumentando o risco de quedas. Também foi possível identificar que grande parte da população apresenta um risco moderado para quedas, servindo de atenção para cuidados preventivos na saúde.

\section{Referências}

ABREU, H.C.A et al. Incidência e fatores preditores de quedas de idosos hospitalizados. CuiabáMT: Rev Saúde Pública, 2015. 49-37 p.

ALVES, A.H.C et al. Ocorrência de quedas entre idosos institucionalizados: prevalência, causas e consequências. João Pessoa-PB: J. Res.: Fundam. Care. Online, 2016. 4376-4386 p. v. 8(2).

ANSAI, J.H et al. Revisão de dois instrumentos clínicos de avaliação para predizer risco de quedas em idosos. Rio de Janeiro: Rev. Bras. Geriatr. Gerontol, 2014. 177-189 p. v. 17(1). 
BRETAN, $\mathrm{O}$ et al. Risk of falling among elderly persons living in the community: assessment by the Timed up and go test. Distrito de Rubião Júnior: Brazilian Journal Of Otorhinolaryngology, 2012. v. 79(1).

COSTA, F.B.S; SOUSA, Brenda Rocha. Teste timed up and go como medida da mobilidade funcional e do equilíbrio em idosos: revisão de literatura. [S.l.]: Revista Eletrônica Acervo Saúde, 2016. 134-140 p. v. 4.

GASPAROTTO, L.P.R ; FALSARELLA, Gláucia Regina ; COIMBRA, Arlete Maria Valente . As quedas no cenário da velhice: conceitos básicos e atualidades da pesquisa em saúde. Rio de Janeiro: Rev. Bras. Geriatr. Gerontol, 2014. 201-209 p.

LEITÃO, M.B et al. Posicionamento Oficial da Sociedade Brasileira de Medicina do Esporte: Atividade Física e Saúde na Mulher. Rio de Janeiro: Rev Bras Med Esporte, 2000. v. 6.

LUZ, E.P da et al. Perfil sociodemográfico e de hábitos de vida da população idosa de um município da região norte do Rio Grande do Sul, Brasil. Rio de Janeiro: Rev. Bras. Geriatr. Gerontol, 2014. 303-314 p. v. 17(2).

MELO, B.R.S; SANTOS, P.R.S; GRATÃO, A.C.M. Fatores de risco extrínsecos para quedas em idosos: uma revisão bibliográfica. [S.1.]: Revista Eletrônica Acervo Saúde, 2014. 695-703 p. v. $6(2)$.

PRESTA, S.A et al. Caracterização e condições de saúde dos idosos do município de passo fundo, no Rio Grande do Sul. [S.1.]: Revista Brasileira de Ciências da Saúde, 2011. v. 29.

PODSIADLO D, RICHARDSON S. The timed "Up \& Go": a test of basic functional mobility for frail elderly persons. J AmGeriatr Soc. 1991;39(2):142-8.

SILVA, C.S et al. Relação dos testes timed up and go e velocidade de marcha nos estágios do envelhecimento. Pampa: Anais do $8^{\circ}$ Salão Internacional de Ensino, Pesquisa e Extensão Universidade Federal do Pampa, 2015.

SILVA, I.A et al. Efeito de um protocolo de Facilitação Neuromuscular Proprioceptiva (FNP) no equilíbrio postural de idosas. [S.1.]: Fisioter Pesqui., 2017. 62-67 p.

WAMSER, E.L et al. Melhor desempenho no teste timed up and go está associado a melhor desempenho funcional em idosas da comunidade. [S.1.]: Geriatr Gerontol Aging, 2013. 138-43 p. v. 9.

\section{Como citar este artigo (Formato ABNT):}

PRADO, Rubiele A.; SANTOS, Kleyton T.; REIS, Luciana A. dos; MORAIS, Karla C.S de; DUTRA Andressa P.; PORTO, Sabrina A. Timed Up and Go em Idosos Residentes na Comunidade. Id on Line Revista Multidisciplinar e de Psicologia, 2017, vol.11, n.38, p.770-777. ISSN: 1981-1179.

Timed Up and Go em Idosos Residentes na Comunidade

Recebido: 08.11.2017

Aceito: 10.11 .2017 